\title{
COMPOSTOS ORGÂNICOS HIDROSSOLÚVEIS DE RESÍDUOS VEGETAIS E SEUS EFEITOS NOS ATRIBUTOS QUÍMICOS DO SOLO $^{(1)}$
}

\author{
Raquel Cátia Diehl ${ }^{(2)}$, Mario Miyazawa ${ }^{(3)} \&$ Hideaki Wilson Takahashi ${ }^{(4)}$
}

\begin{abstract}
RESUMO
Compostos orgânicos hidrossolúveis de resíduos vegetais depositados na superfície do solo podem melhorar a fertilidade do subsolo, pela neutralização da acidez e transporte de $\mathrm{Ca}$ e $\mathrm{Mg}$. Com o objetivo de avaliar o efeito dos compostos orgânicos hidrossolúveis de materiais vegetais nos atributos químicos de um Latossolo Vermelho distroférrico, foi desenvolvido um experimento no Instituto Agronômico do Paraná (IAPAR), Londrina, com amostras de solo acondicionadas em colunas nas quais se aplicaram os tratamentos: água destilada, calcário incorporado na camada 0-5 cm de profundidade, calcário e percolação com extratos de nabo forrageiro, aveia preta, palha de trigo, milho e soja. No extrato percolado, foram determinados os teores de ligantes orgânicos hidrossolúveis (LOH) por potenciometria com eletrodo seletivo de $\mathrm{Cu}^{2+}$; ácidos orgânicos tituláveis (AOT) por titulação ácido-base e ânions orgânicos (AO) pela soma de bases. As concentrações de AO e AOT variaram de 7,0 a 32,0 $\mathrm{mmol} \mathrm{L}^{-1}$ e de $\mathrm{LOH}$ de 0,60 a 2,23 mmol L-1. Todos os extratos vegetais aumentaram o $\mathrm{pH}$, os teores de $\mathrm{Ca}, \mathrm{Mg}$ e $\mathrm{K}$ trocável e diminuíram a acidez potencial e o $\mathrm{Al}$ trocável até $15 \mathrm{~cm}$ de profundidade, enquanto o efeito da calagem sem extrato foi observado somente até $10 \mathrm{~cm}$ de profundidade. A concentração de compostos orgânicos solúveis oriundos dos materiais vegetais correlacionou-se com o $\mathrm{pH}, \mathrm{Al}$ trocável, $\mathrm{H}+\mathrm{Al}$ e V \% do solo na camada de $\mathbf{0 - 2 0} \mathrm{cm}$, confirmando a participação destes na melhoria dos atributos químicos do solo e ação da calagem superficial quando o material vegetal está presente.
\end{abstract}

Termos de indexação: calcário; ligante orgânico; acidez; cátions básicos, adubo verde.

\footnotetext{
(1) Trabalho apresentado no XXXI Congresso Brasileiro de Ciência do Solo, 2007 (Gramado, RS).

(2) Mestre em Agronomia pelo Programa de Pós-Graduação em Agronomia, Universidade Estadual de Londrina (UEL), Rodovia Celso Garcia Cid, PR 445 Km 380 Campus Universitário CEP: 86051-990, Londrina (PR). E-mail: rdiehl@milenia.com.br

(3) Pesquisador do Instituto Agronômico do Paraná (IAPAR), Rodovia Celso Garcia Cid, Km 375, Caixa Postal 131, CEP: 86001-970 Londrina (PR). E-mail: miyazawa@iapar.br

(4) Professor Adjunto do Programa de Pós-Graduação em Agronomia - UEL. E-mail: hwilson@uel.br
} 


\title{
SUMMARY: WATER-SOLUBLE ORGANIC COMPOUNDS IN PLANT RESIDUE AND THE EFFECTS ON SOIL CHEMICAL PROPERTIES
}

\begin{abstract}
The water-soluble organic compounds of plant residues released on the soil surface can improve the subsoil fertility, due to the neutralization of acidity and $\mathrm{Ca}$ and $\mathrm{Mg}$ transport. An experiment was conducted at the Instituto Agronomico do Parana (IAPAR), Londrina, to evaluate the effect of water-soluble organic compounds of plant extracts on the chemical characteristics of a dark Red Latosol (Typic Haplortox), on soil samples in columns. The treatments consisted of: distilled water, lime incorporated in the $0-5 \mathrm{~cm}$ soil layer, lime and percolation of the following plant extracts: oilseed radish, black oat and wheat, maize and soybean straw. The following properties were determined in the plant extracts: the water-soluble organic ligand (WSOL) by potentiometry with selective $\mathrm{Cu}^{2+}$ electrode; titration of organic anions (TOA) and organic anions (OA) by base addition. The $O A$ and TOA concentrations varied from 7.0 to $32.0 \mathrm{mmol} \mathrm{L}^{-1}$ and WSOL from 0.60 to $2.23 \mathrm{mmol} \mathrm{L}$ 1. All plant extracts increased $\mathrm{pH}$ and exchangeable $\mathrm{Ca}, \mathrm{Mg}$ and $\mathrm{K}$ and decreased the potential acidity and exchangeable Al down to a soil depth of $15 \mathrm{~cm}$, while the effect of lime without plant extract was only observed down to $10 \mathrm{~cm}$. The soluble organic compound concentrations of the plant residues were correlated with $\mathrm{pH}, \mathrm{Al}^{3+}, \mathrm{H}+\mathrm{Al}$ and soil base saturation in the $0-20 \mathrm{~cm}$ layer, confirming the role of these organic compounds to improve the chemical characteristics of an acid soil as well as the action of the surface-applied lime.
\end{abstract}

Index terms: lime; organic ligand; soil acidity; basic cations; green manure.

\section{INTRODUÇÃO}

Compostos orgânicos de baixo peso molecular são comumente encontrados em solos, cultivados ou não, originários, principalmente, da exsudação de fungos e raízes de plantas (Jones, 1998) e da decomposição e solubilização da matéria orgânica depositada na superfície ou incorporada nos horizontes superficiais do solo (Devêvre et al., 1996). Esses compostos orgânicos apresentam grupos funcionais que participam de várias reações químicas no solo, como complexação metal-orgânica (Hue et al., 1986) e adsorção de íons, podendo promover a amenização da toxidez do Al trocável, mobilização do calcário e de cátions no perfil do solo (Pavan \& Miyazawa, 1998; Ziglio et al., 1999).

O efeito da calagem, em curto prazo, é restrito, principalmente, nas proximidades do local de aplicação (Gonzalez-Enrico et al., 1979). Para Oliveira \& Pavan (1996), a presença de resíduos vegetais na superfície do solo sob plantio direto constitui a principal causa da eficiência da calagem na correção da acidez subsuperficial do solo. Cassiolato et al., (2000) relataram que o efeito do resíduo vegetal na mobilidade do calcário no solo varia com a espécie da planta e com as variedades dentro de uma mesma espécie.

Embora reconhecido que compostos liberados do material vegetal promovam alterações químicas em solos sob sistema plantio direto, a avaliação desses efeitos está limitada pela determinação analítica e quantificação dos compostos orgânicos, pelos custos, praticidade e eficácia. A estimativa dos compostos orgânicos solúveis na solução do solo, baseada na capacidade de complexação dos ligantes orgânicos hidrossolúveis com os íons metálicos, juntamente com a análise de propriedades químicas, como condutividade, comportamento ácido-base, concentração de metais no extrato vegetal, e a relação desses com atributos químicos do solo, são necessárias para avaliar o efeito do material vegetal nas alterações químicas do solo sob sistema plantio direto em condição de campo.

Nesse contexto, este estudo foi desenvolvido com os objetivos de: a) estimar os compostos orgânicos hidrossolúveis em extratos vegetais; $b$ ) avaliar o efeito destes extratos, na presença da calagem superficial, nos atributos químicos do perfil do solo, e c) verificar a relação dos compostos orgânicos solúveis, estimados por potenciometria, titulação ácido-base e soma de bases, com as alterações químicas do solo causadas pela aplicação de extratos vegetais associados à calagem.

\section{MATERIAL E MÉTODOS}

O experimento foi realizado em condições de laboratório, no Instituto Agronômico do Paraná IAPAR/Londrina/PR. Uma amostra do horizonte Bw de um Latossolo Vermelho distroférrico argiloso foi coletada em área não cultivada, onde uma subamostra foi retirada para análise química do $\mathrm{pH}$ em $\mathrm{CaCl}_{2}(4,2)$; 
$\mathrm{Al}$ trocável $\left(1,51 \mathrm{cmol}_{\mathrm{c}} \mathrm{dm}^{-3}\right) ; \mathrm{H}+\mathrm{Al}\left(9,0 \mathrm{cmol}_{c} \mathrm{dm}^{-3}\right)$; Ca trocável $\left(0,76 \mathrm{cmol}_{\mathrm{c}} \mathrm{dm}^{-3}\right), \mathrm{Mg}$ trocável $(0,29$ $\left.\mathrm{cmol}_{\mathrm{c}} \mathrm{dm}^{-3}\right)$; K trocável $\left(0,16 \mathrm{cmol}_{\mathrm{c}} \mathrm{dm}^{-3}\right)$ e C orgânico $\left(17,0 \mathrm{~g} \mathrm{dm}^{-3}\right)$, determinados conforme descrito em Pavan et al. (1992). O solo restante foi seco ao ar, moído e passado em peneira de $2 \mathrm{~mm}$ para posterior instalação do experimento.

Amostras de nabo forrageiro (Raphanus sativus) e aveia preta (Avena strigosa) foram coletadas no estádio de pleno florescimento; trigo (Triticum aestivum), palha de milho (Mea zays) e palha de soja (Glycine max) após a colheita dos grãos em área experimental do IAPAR. As amostras dos materiais vegetais foram secas em estufa a $65^{\circ} \mathrm{C}$, durante $48 \mathrm{~h}$, moídas, passadas em peneira de $1 \mathrm{~mm}$ e armazenadas em sacos de papel. O extrato vegetal utilizado para estimativa dos compostos hidrossolúveis nos materiais vegetais foi obtido na proporção de 1:50 (material vegetal: água destilada). As soluções foram agitadas por quatro horas a 250 rpm e filtradas em papel filtro quantitativo.

A estimativa dos compostos hidrossolúveis dos extratos vegetais foi realizada pelas seguintes técnicas:

a) Ligantes Orgânicos Hidrossolúveis (LOH): os ligantes orgânicos no extrato vegetal foram estimados por potenciometria utilizando eletrodo seletivo de íon $\mathrm{Cu}^{2+}$. O princípio do método foi baseado na complexação do $\mathrm{Cu}^{2+}$ com os ligantes orgânicos dos resíduos vegetais. Um excesso de $\mathrm{Cu}^{2+}$ é adicionado à amostra e, após um tempo de equilíbrio determina-se o $\mathrm{Cu}^{2+}$ livre, admitindo-se que a diferença entre a concentração inicial e final de $\mathrm{Cu}^{2+}$ corresponda à concentração de ligantes orgânicos em solução. A curva analítica do $\mathrm{Cu}^{2+}$ foi preparada em solução-tampão com NaOAc 1,0 mol L-1 $+\mathrm{KNO}_{3}$ $1,0 \mathrm{~mol} \mathrm{~L}^{-1}, \mathrm{pH} 4,5$ nas seguintes concentrações: 0,0; 0,$1 ; 0,2 ; 0,5 ; 1,0 ; 2,04,0$ e $5,0 \times 10^{-4} \mathrm{~mol} \mathrm{~L}^{-1}$. Para a análise das amostras, transferiu-se uma alíquota de $5,0 \mathrm{~mL}$ do extrato vegetal para tubo de ensaio de 30 $\mathrm{mL} ; 10 \mathrm{~mL}$ de solução-tampão com NaOAc $2,0 \mathrm{~mol}$ $\mathrm{L}^{-1}+\mathrm{KNO}_{3} 2,0 \mathrm{~mol} \mathrm{~L}^{-1}$ e $5,0 \mathrm{~mL}$ de $\mathrm{Cu}^{2+} 2,0 \times 10^{-3}$ mol L-1, efetuando-se a leitura no potenciômetro 30 min após homogeneização.

b) Ácidos Orgânicos Tituláveis (AOT): os ácidos orgânicos foram estimados pela titulação potenciométrica em intervalo de $\mathrm{pH}$ 3,0 e 7,0. Esse intervalo foi utilizado admitindo-se que no pH 3,0 a maioria dos ânions orgânicos está na forma protonada, enquanto, no $\mathrm{pH}$ 7,0, estaria na forma ionizada (Young et al., 1981). Transferiram-se $25 \mathrm{~mL}$ da amostra para copo de 150 $\mathrm{mL}$, adicionaram-se $25 \mathrm{~mL}$ de $\mathrm{H}_{2} \mathrm{O}$, sendo o excesso de solução de $\mathrm{HCl} 2,0 \mathrm{~mol} \mathrm{~L}^{-1}$ titulada com solução de $\mathrm{NaOH} 0,1 \mathrm{~mol} \mathrm{~L}^{-1}$ padronizada, do $\mathrm{pH}$ 3,0 até pH 7,0.

c) Ânions Orgânicos (AO): os ânions orgânicos foram estimados indiretamente pela soma dos cátions Ca, Mg e K (Franchini et al., 2001), sendo o Ca e o Mg determinados por emissão atômica induzida por plasma e K por fotometria de chama. Todas as determinações foram realizadas em triplicatas.
Foram preparadas colunas de PVC com diâmetro interno de $3,0 \mathrm{~cm}$ por $50,0 \mathrm{~cm}$ de altura, revestidas por um saco plásticos, que continha algodão sintético inerte na parte inferior da coluna. As colunas foram preenchidas até $40 \mathrm{~cm}$ de altura com o solo sob vibração até densidade aparente aproximada de $1,2 \mathrm{~kg} \mathrm{dm}^{-3}$, o que correspondeu a $340 \mathrm{~g}$ de solo e determinou-se volume de poros, por meio da saturação com água, cujo volume correspondente foi de $165 \mathrm{~mL}$. Previamente à instalação do experimento, uma amostra de solo foi incubada com calcário durante 21 dias com umidade na capacidade de campo. A dose de calcário dolomítico (preparado a partir de $\mathrm{CaCO}_{3} \mathrm{e}$ $\mathrm{MgCO}_{3}$, qualidade técnica, relação 1:1) foi em quantidade equivalente para neutralizar $100 \%$ da acidez potencial $(\mathrm{H}+\mathrm{Al})$ do solo. $\mathrm{O}$ solo previamente incubado substituiu a camada de $0-5 \mathrm{~cm}$ das colunas, excluindo a testemunha, simulando a aplicação superficial do calcário em sistema plantio direto.

Os seguintes tratamentos foram aplicados na superfície do solo condicionado nas colunas de PVC: a) água destilada (testemunha); b) calcário sem extrato vegetal; c) calcário + extratos de nabo forrageiro; d) aveia preta; e) palha de trigo;f) palha de milho; g) palha de soja. O extrato vegetal foi obtido pela adição de $165 \mathrm{~mL}$ de água destilada em 1,4 g do material vegetal (equivalente a $20 \mathrm{t} \mathrm{ha}^{-1}$ ), agitado a $250 \mathrm{rpm}$, durante quatro horas, e filtrado, sendo adicionado na superfície do solo, assim como a água destilada na testemunha, na vazão de $3,45 \mathrm{~mL} \mathrm{~h}^{-1}$, em dois dias. Após a adição dos extratos, as colunas de solo foram separadas em camadas de $0-5 ; 5-10$; $10-15 ; 15-20 ; 20-30 ; 30-40 \mathrm{~cm}$, secas em estufa a $60{ }^{\circ} \mathrm{C}$ e determinado o $\mathrm{pH}$ em $\mathrm{CaCl}_{2} ; \mathrm{H}+\mathrm{Al} ; \mathrm{Al}^{3+} ; \mathrm{Ca}^{2+}$; $\mathrm{Mg}^{2+} ; \mathrm{K}^{2+} \mathrm{e} \mathrm{V} \%$.

O delineamento utilizado no experimento foi o de blocos inteiramente casualizados, com três repetições, em parcelas subdivididas, alocando-se os tratamentos aplicados em superfície como fator principal, e a profundidade de amostragem do solo, como subfator. Os resultados foram submetidos à análise de variância e a diferença entre médias de tratamentos foi avaliada pelo teste de Tukey a $5 \%$.

\section{RESULTADOS E DISCUSSÃO}

$\mathrm{O} \mathrm{pH}$ e os teores dos cátions básicos solúveis nos extratos vegetais estão apresentados no quadro 1 . As maiores concentrações foram encontradas nos extratos do nabo forrageiro e aveia preta e as menores nas culturas do trigo, milho e soja. No quadro 2 , estão indicadas as concentrações de ligantes orgânicos, ácidos orgânicos tituláveis e ânions orgânicos nos extratos vegetais. Os resultados mostram que as concentrações de compostos orgânicos variaram conforme as espécies vegetais e apresentaram estreita correlação entre as concentrações estimadas pelas diferentes técnicas $\left(p<0,01 R^{2}=0,95\right)$. 
Quadro 1. Valor de pH e teores de K, Ca e Mg hidrossolúveis nos extratos dos resíduos vegetais

\begin{tabular}{lrrrr}
\hline Resíduo vegetal & $\mathbf{p H}$ & $\mathbf{K}$ & $\mathbf{C a}$ & $\mathbf{M g}$ \\
\hline & \multicolumn{4}{c}{ mmol $_{\mathrm{c}} \mathrm{L}^{-1}$} \\
Nabo forrageiro & 6,20 & 23,00 & 15,00 & 4,60 \\
Aveia preta & 7,20 & 17,90 & 3,40 & 2,20 \\
Trigo & 5,60 & 10,70 & 1,40 & 1,20 \\
Milho & 5,80 & 6,00 & 0,40 & 3,00 \\
Soja & 5,80 & 5,80 & 0,60 & 2,00 \\
\hline
\end{tabular}

Quadro 2. Valor de teores de ligantes orgânicos hidrossolúveis (LOH), ácidos orgânicos tituláveis (AOT) e ânions orgânicos (AO) nos extratos vegetais

\begin{tabular}{lccc}
\hline Resíduo vegetal & LOH(1) $^{(1)}$ & AOT $^{(2)}$ & AO $^{(3)}$ \\
\hline & \multicolumn{2}{c}{$\mathrm{mmol} \mathrm{L}^{-1}$} & $\mathrm{mmol}_{\mathrm{c}} \mathrm{L}^{-1}$ \\
Nabo forrageiro & 2,23 & 23,71 & 42,60 \\
Aveia preta & 1,76 & 18,50 & 23,50 \\
Trigo & 1,30 & 12,40 & 13,00 \\
Milho & 0,76 & 10,50 & 9,40 \\
Soja & 0,60 & 9,00 & 8,40 \\
\hline
\end{tabular}

${ }^{(1)}$ Potenciometria; ${ }^{(2)}$ Titulação ácido-base; ${ }^{(3)} \varepsilon$ Bases.

O grupo LOH corresponde aos compostos orgânicos que formam complexos com metais polivalentes $\left(\mathrm{Al}^{3+}, \mathrm{Ca}^{2+}\right.$ e $\left.\mathrm{Mg}^{2+}\right)$ e apresentam constante de estabilidade média e, ou, alta, como os ânions citrato, oxalato, tartarato e malato. Outros ânions orgânicos podem estar presentes em solução, mas, por formarem complexos com constante de estabilidade baixa com o $\mathrm{Cu}^{2+}$, não são detectados por esta técnica. O grupo AOT, além dos mesmos compostos estimados pela técnica potenciométrica, corresponde a todos os compostos com grupamentos ácidos que apresentam comportamento ácido-base, podendo ser titulados com uma base forte como o $\mathrm{NaOH}$. Nesse grupo de compostos, estão também incluídos ácidos que formam compostos de baixa estabilidade com os cátions metálicos polivalentes do extrato vegetal, tais como os ácidos: fórmico acético e succínico. O grupo AO, estimado indiretamente por meio da soma de bases corresponde à concentração de ácidos orgânicos no extrato vegetal, visto que, no tecido foliar, esses compostos estão presentes na forma de ânions orgânicos para a manutenção da eletroneutralidade química, como resultado da absorção de cátions. Os resultados podem ser subestimados pela presença de ânions inorgânicos, como o $\mathrm{Cl}^{-}, \mathrm{SO}_{4}{ }^{2-}$ e $\mathrm{NO}_{3}{ }^{-}$. Entretanto, conforme verificado neste trabalho, os resultados de $\mathrm{AO}$ e AOT foram similares, exceto para o extrato de nabo forrageiro, indicando que a presença desses ânions inorgânicos no extrato vegetal é baixa.
Conforme observado por Franchini et al., (2001), ocorre redução nas concentrações de $\mathrm{Ca}, \mathrm{Mg}$ e $\mathrm{Ke}$, conseqüentemente, dos compostos orgânicos hidrossolúveis com o avanço da idade da planta. Como os resíduos das culturas comerciais são deixados no solo após a colheita do grão, ou seja, em estádio fenológico bem avançado, os teores de ligantes orgânicos e compostos solúveis são bem menores, conforme constatado neste estudo.

Para melhor visualização dos resultados dos atributos químicos ao longo da coluna de solo nos diferentes tratamentos, foram omitidos os dados referentes ao tratamento milho e soja, considerando o tratamento trigo como representativo dos materiais com baixos teores de compostos orgânicos (Quadro 3). A adição do calcário, sem extratos vegetais, promoveu alterações significativas no $\mathrm{pH}$ apenas na camada superficial $(0-5 \mathrm{~cm})$, onde o calcário foi incorporado. Esses resultados demonstram a baixa eficácia do calcário aplicado em superfície para reduzir a acidez subsuperficial dos solos, corroborando os resultados obtidos por Moreira et al., 2001. Em contraste, a adição dos extratos vegetais mais calagem promoveu aumento significativo no $\mathrm{pH}$ até à camada de $10-15 \mathrm{~cm}$, em todos os tratamentos, sendo esse aumento efetivo até à camada de $15-20 \mathrm{~cm}$ para a aveia e até $30 \mathrm{~cm}$ para o nabo. $\mathrm{O}$ aumento do $\mathrm{pH}$ na presença dos extratos vegetais e a extensão dos efeitos corretivos em profundidade estão relacionados com o poder alcalinizante que estes materiais apresentam (Quadro 1). Resultados semelhantes foram relatados por Cassiolato et al., (2000) em um Latossolo Vermelho-Escuro, com aumentos de $\mathrm{pH}$ até $10 \mathrm{~cm}$, quando o calcário foi aplicado em superfície, e até $20 \mathrm{~cm}$, quando extratos de aveia foram aplicados junto com a calagem. Meda et al., (2001) verificaram aumento do $\mathrm{pH}$ até $20 \mathrm{~cm}$ de profundidade com aplicação de extratos de tremoço sem calcário em um solo ácido.

O Al trocável foi totalmente neutralizado na camada onde o calcário foi incorporado e reduzido a $1,33 \mathrm{cmol}_{\mathrm{c}}$ $\mathrm{dm}^{-3}$ na camada de $5-10 \mathrm{~cm}$, sem efeitos nas demais camadas, sendo o mesmo observado para o $\mathrm{H}+\mathrm{Al}$, tendo seu valor reduzido em cerca de $30 \%$ na camada de 5 $10 \mathrm{~cm}$, sem alterar as demais camadas. Na presença dos extratos vegetais, o extrato de trigo reduziu significativamente o $\mathrm{Al}$ trocável até $15 \mathrm{~cm}$ e o $\mathrm{H}+\mathrm{Al}$ até à camada de $15-20 \mathrm{~cm}$. A aveia e o nabo foram eficientes na diminuição do $\mathrm{Al}$ até $40 \mathrm{~cm}$, sendo totalmente neutralizados até à camada de $5-10 \mathrm{~cm}$. Para o $\mathrm{H}+\mathrm{Al}$, o efeito significativo da aveia foi observado até $30 \mathrm{~cm}$ e com o nabo até $40 \mathrm{~cm}$, tendo este aumentado em cerca de $23 \%$ a ação corretiva da calagem na camada de 0-5 cm. Miyazawa et al., (2002) observaram redução do Al trocável até $10 \mathrm{~cm}$ em um solo ácido na presença de calagem mais extrato de trigo e até $50 \mathrm{~cm}$ com extrato de aveia preta. Nas camadas superficiais $(0-10$ $\mathrm{cm}$ ), ambos os extratos promoveram redução do $\mathrm{H}+\mathrm{Al}$ a valores próximos a calagem, sendo o nabo mais eficiente que a calagem em todas as camadas. Liu \& Hue (1996) verificaram que o efeito do calcário aplicado em superfície na saturação por $\mathrm{Al}$ ficou restrito a 10 $\mathrm{cm}$ de profundidade, enquanto a aplicação de Ca-fulvato derivado de materiais orgânicos promoveu redução até à camada de $45-50 \mathrm{~cm}$. 
Quadro 3. Valores de pH e teores de Al trocável, H+Al, Ca, Mg e K trocáveis em diferentes profundidades da coluna de solos, após percolação de extratos vegetais, na presença de calagem

\begin{tabular}{|c|c|c|c|c|c|}
\hline Prof. & Testemunha & Calcário & Aveia & Trigo & Nabo \\
\hline $\mathrm{cm}$ & & & $\mathrm{pH}$ & & \\
\hline $0-5$ & $4,20 \mathrm{aD}$ & $5,69 \mathrm{aC}$ & $6,70 \mathrm{aB}$ & $6,48 \mathrm{aB}$ & 7,17 aA \\
\hline $5-10$ & $4,20 \mathrm{aE}$ & $4,40 \mathrm{bD}$ & $5,50 \mathrm{bAC}$ & $5,15 \mathrm{bB}$ & $5,57 \mathrm{bA}$ \\
\hline $10-15$ & $4,20 \mathrm{aD}$ & $4,20 \mathrm{bD}$ & $4,97 \mathrm{cAC}$ & $4,55 \mathrm{cB}$ & $5,04 \mathrm{cA}$ \\
\hline $15-20$ & $4,20 \mathrm{aC}$ & $4,20 \mathrm{bC}$ & $4,50 \mathrm{~dB}$ & $4,20 \mathrm{dC}$ & $4,92 \mathrm{cA}$ \\
\hline $20-30$ & $4,20 \mathrm{aB}$ & $4,20 \mathrm{bB}$ & $4,20 \mathrm{eB}$ & $4,20 \mathrm{~dB}$ & $4,33 \mathrm{dA}$ \\
\hline \multirow[t]{2}{*}{$30-40$} & $4,20 \mathrm{aA}$ & $4,20 \mathrm{bA}$ & $4,20 \mathrm{eA}$ & $4,20 \mathrm{dA}$ & $4,20 \mathrm{dA}$ \\
\hline & \multicolumn{5}{|c|}{$\mathrm{Al}^{3+}\left(\mathrm{cmol}_{\mathrm{c}} \mathrm{dm}^{-3}\right)$} \\
\hline $0-5$ & $1,50 \mathrm{aA}$ & $0,00 \mathrm{cB}$ & $0,00 \mathrm{eB}$ & $0,00 \mathrm{~dB}$ & $0,00 \mathrm{eB}$ \\
\hline $5-10$ & $1,50 \mathrm{aA}$ & $1,33 \mathrm{bB}$ & $0,00 \mathrm{eD}$ & $0,35 \mathrm{cC}$ & $0,00 \mathrm{eD}$ \\
\hline $10-15$ & $1,51 \mathrm{aA}$ & $1,53 \mathrm{aA}$ & $0,25 \mathrm{dC}$ & $0,80 \mathrm{bB}$ & $0,20 \mathrm{dC}$ \\
\hline $15-20$ & $1,51 \mathrm{aA}$ & $1,51 \mathrm{aA}$ & $1,15 \mathrm{bcC}$ & $1,47 \mathrm{aB}$ & $0,65 \mathrm{cD}$ \\
\hline $20-30$ & $1,52 \mathrm{aA}$ & $1,51 \mathrm{aA}$ & $1,20 \mathrm{bB}$ & $1,52 \mathrm{aA}$ & $0,95 \mathrm{bC}$ \\
\hline \multirow[t]{2}{*}{$30-40$} & $1,52 \mathrm{aA}$ & $1,52 \mathrm{aA}$ & $1,40 \mathrm{aA}$ & $1,52 \mathrm{aA}$ & $1,40 \mathrm{aA}$ \\
\hline & \multicolumn{5}{|c|}{$\mathrm{H}+\mathrm{Al}\left(\mathrm{cmol}_{\mathrm{c}} \mathrm{dm}^{-3}\right)$} \\
\hline $0-5$ & $9,00 \mathrm{aA}$ & $4,28 \mathrm{cB}$ & $3,18 \mathrm{dC}$ & $3,18 \mathrm{eC}$ & $2,16 \mathrm{fD}$ \\
\hline $5-10$ & 8,99 aA & $6,24 \mathrm{bB}$ & $3,19 \mathrm{dD}$ & $4,23 \mathrm{dC}$ & $3,43 \mathrm{eD}$ \\
\hline $10-15$ & $8,99 \mathrm{aA}$ & $8,99 \mathrm{aA}$ & $4,50 \mathrm{cC}$ & $6,68 \mathrm{cB}$ & $4,50 \mathrm{dC}$ \\
\hline $15-20$ & $9,00 \mathrm{aA}$ & $8,98 \mathrm{aA}$ & $6,47 \mathrm{bC}$ & $7,75 \mathrm{bB}$ & $5,81 \mathrm{cD}$ \\
\hline $20-30$ & $9,00 \mathrm{aA}$ & 8,99 aA & $6,70 \mathrm{bB}$ & 8,98 aA & $6,72 \mathrm{bB}$ \\
\hline \multirow[t]{2}{*}{$30-40$} & $9,00 \mathrm{aA}$ & $9,00 \mathrm{aA}$ & 8,99 aA & 8,99 aA & $8,76 \mathrm{aA}$ \\
\hline & \multicolumn{5}{|c|}{$\mathrm{Ca}^{2+}\left(\mathrm{cmol}_{\mathrm{c}} \mathrm{dm}^{-3}\right)$} \\
\hline $0-5$ & $0,75 \mathrm{aE}$ & $4,53 \mathrm{aBC}$ & $4,66 \mathrm{aB}$ & $4,31 \mathrm{aC}$ & $5,46 \mathrm{aA}$ \\
\hline $5-10$ & $0,74 \mathrm{aD}$ & $1,18 \mathrm{bC}$ & $2,21 \mathrm{bB}$ & $2,06 \mathrm{bB}$ & $3,14 \mathrm{bA}$ \\
\hline $10-15$ & $0,75 \mathrm{aE}$ & $1,10 \mathrm{bD}$ & $1,62 \mathrm{cB}$ & $1,34 \mathrm{cC}$ & $2,18 \mathrm{cA}$ \\
\hline $15-20$ & $0,76 \mathrm{aC}$ & $0,78 \mathrm{cC}$ & $1,16 \mathrm{~dB}$ & $1,18 \mathrm{cB}$ & $1,45 \mathrm{dA}$ \\
\hline $20-30$ & $0,77 \mathrm{aC}$ & $0,79 \mathrm{cC}$ & $0,96 \mathrm{eB}$ & $0,79 \mathrm{dC}$ & $1,31 \mathrm{deA}$ \\
\hline \multirow[t]{2}{*}{$30-40$} & $0,76 \mathrm{aB}$ & $0,79 \mathrm{cB}$ & $0,79 \mathrm{eB}$ & $0,79 \mathrm{~dB}$ & $1,20 \mathrm{eA}$ \\
\hline & \multicolumn{5}{|c|}{$\mathrm{Mg}^{2+}\left(\mathrm{cmol}_{\mathrm{c}} \mathrm{dm}^{-3}\right)$} \\
\hline $0-5$ & $0,30 \mathrm{aE}$ & $3,40 \mathrm{aA}$ & $2,87 \mathrm{aC}$ & $3,14 \mathrm{aB}$ & $2,62 \mathrm{aD}$ \\
\hline $5-10$ & $0,29 \mathrm{aE}$ & $0,47 \mathrm{bD}$ & $1,41 \mathrm{bB}$ & $1,10 \mathrm{bC}$ & $1,64 \mathrm{bA}$ \\
\hline $10-15$ & $0,28 \mathrm{aC}$ & $0,40 \mathrm{bC}$ & $0,80 \mathrm{cB}$ & $0,75 \mathrm{cB}$ & $0,97 \mathrm{cA}$ \\
\hline $15-20$ & $0,29 \mathrm{aC}$ & $0,31 \mathrm{bC}$ & $0,62 \mathrm{cB}$ & $0,51 \mathrm{~dB}$ & $0,82 \mathrm{cA}$ \\
\hline $20-30$ & $0,29 \mathrm{aB}$ & $0,31 \mathrm{bB}$ & $0,33 \mathrm{~dB}$ & $0,30 \mathrm{eB}$ & $0,64 \mathrm{dA}$ \\
\hline \multirow[t]{2}{*}{$30-40$} & $0,30 \mathrm{aA}$ & $0,31 \mathrm{bA}$ & $0,30 \mathrm{dA}$ & $0,30 \mathrm{eA}$ & $0,39 \mathrm{eA}$ \\
\hline & \multicolumn{5}{|c|}{$\mathrm{K}^{2+}\left(\mathrm{cmol}_{\mathrm{c}} \mathrm{dm}^{-3}\right)$} \\
\hline $0-5$ & $0,13 \mathrm{aD}$ & 0,19 a $\mathrm{D}$ & $3,27 \mathrm{aB}$ & $3,09 \mathrm{aBC}$ & $3,92 \mathrm{aA}$ \\
\hline $5-10$ & $0,14 \mathrm{aE}$ & $0,18 \mathrm{aE}$ & $1,40 \mathrm{bD}$ & $2,39 \mathrm{bB}$ & $2,99 \mathrm{bA}$ \\
\hline $10-15$ & $0,16 \mathrm{aD}$ & $0,20 \mathrm{aD}$ & $1,05 \mathrm{cB}$ & $0,63 \mathrm{cC}$ & $1,37 \mathrm{cA}$ \\
\hline $15-20$ & $0,17 \mathrm{aB}$ & $0,18 \mathrm{aB}$ & $0,23 \mathrm{~dB}$ & $0,19 \mathrm{~dB}$ & $0,62 \mathrm{dA}$ \\
\hline $20-30$ & $0,19 \mathrm{aA}$ & $0,19 \mathrm{aA}$ & $0,20 \mathrm{dA}$ & $0,19 \mathrm{dA}$ & $0,27 \mathrm{eA}$ \\
\hline $30-40$ & $0,19 \mathrm{aA}$ & $0,19 \mathrm{aA}$ & $0,20 \mathrm{dA}$ & $0,19 \mathrm{dA}$ & $0,21 \mathrm{eA}$ \\
\hline
\end{tabular}

Médias seguidas de mesmas letras minúsculas na coluna e maiúsculas na linha não diferem significativamente pelo Teste de Tukey $\mathrm{p}<0,05$. 
$\mathrm{Na}$ ausência dos extratos vegetais, a calagem promoveu aumento significativo nos teores de Ca e $\mathrm{Mg}$ trocáveis até à camada de 5-10 cm (Quadro 3), embora o efeito tenha sido mais pronunciado na camada de $0-5 \mathrm{~cm}$, corroborando os resultados encontrados por Caires et al., (2003) que relataram efeito da calagem em superfície no aumento dos teores de $\mathrm{Ca}$ e $\mathrm{Mg}$ principalmente na camada superficial. Conforme relatado por Liu \& Hue (1996), cargas negativas no complexo de troca geradas pelo aumento do $\mathrm{pH}$ inerente à calagem são responsáveis pelo aumento destes cátions na camada onde o calcário foi incorporado, considerando este como fonte de Ca e Mg. Nos tratamentos extratos vegetais mais calagem, o comportamento em profundidade foi diferenciado entre as espécies: o extrato de trigo promoveu aumento significativo desses cátions até à camada de $15-20 \mathrm{~cm}$, enquanto a aveia até $30 \mathrm{~cm}$ e novamente o nabo apresentaram os maiores efeitos que se estenderam até à camada de 30 $-40 \mathrm{~cm}$, demonstrando o potencial desses materiais em mobilizar cátions polivalentes. Meda et al., (2001) também relataram aumentos do Ca trocável na presença de extratos de aveia até $10 \mathrm{~cm}$ de profundidade, enquanto o extrato de tremoço promoveu alterações até à camada de $20-25 \mathrm{~cm}$. Corroborando os resultados encontrados no presente estudo, maior efeito do nabo em relação à aveia na mobilização do Ca foi verificado por Amaral et al., (2004) até à camada de 15-20 cm.

Com relação ao K trocável, a aplicação de calcário não promoveu alterações no solo, uma vez que nenhuma fonte desse elemento foi fornecida, sendo na testemunha observado pequeno aumento nas profundidades maiores, indicativo da lixiviação do $\mathrm{K}$ presente no complexo de troca pela água percolada. Nos tratamentos que continham extratos vegetais, todos promoveram aumentos significativos até à camada de $10-15 \mathrm{~cm}$, sendo o efeito do nabo observado até à camada de $20-30 \mathrm{~cm}$. Os aumentos dos cátions básicos no solo na presença dos extratos vegetais foram relacionados com o teor desses cátions na solução inicial (Quadro 1), onde o nabo apresentava o maior teor, tanto de Ca como de $\mathrm{Mg}$ e K. No entanto, inerente a todos os extratos, observou-se o acúmulo de K na superfície com diminuição do seu teor em profundidade. Miyazawa et al., (2002), em estudo realizado em colunas de PVC com amostras de um Latossolo Vermelho ácido, semelhante ao do presente estudo, verificaram que extratos vegetais aplicados com calcário aceleraram os transportes de $\mathrm{Ca}$ e $\mathrm{Mg}$ em profundidade, enquanto o $\mathrm{K}$ teve seus teores aumentados nas camadas superficiais do solo, notadamente com o resíduo de aveia preta.

Como conseqüência do aumento dos cátions básicos e diminuição da acidez do solo, a saturação por bases aumentou na presença dos extratos vegetais e da calagem (dados não apresentados). A aplicação de calcário elevou a saturação por bases a $65,5 \%$ na camada onde ele foi incorporado, sendo drasticamente reduzida na camada posterior (22,7\%) e sem alterações significativas nas demais camadas. A aplicação dos extratos, independentemente do material vegetal, foi efetiva no aumento da saturação por bases acima de $70 \%$ na camada de $0-5 \mathrm{~cm}$. O efeito do resíduo de trigo foi observado até à camada de $15-20 \mathrm{~cm}$ e a aveia até $20-30 \mathrm{~cm}$, enquanto o nabo promoveu aumentos até 40 $\mathrm{cm}$. O rápido aumento da saturação por bases na presença dos resíduos vegetais pode ser vantajoso em relação à calagem isolada, considerando que a ação do calcário aplicado superficialmente é limitada pela lenta taxa de dissolução do $\mathrm{CaCO}_{3}$ (Pavan, 1994) e seu efeito na redução da acidez do subsolo depende da lixiviação de sais inorgânicos e, ou, orgânicos por meio do perfil do solo.

As alterações nas características químicas do solo correlacionaram-se com a concentração de LOH, AOT e AO nos extratos vegetais (Quadro 4), demonstrando que tais alterações foram promovidas

Quadro 4. Equações de regressão e coeficientes de determinação entre atributos químicos do solo (y) e as concentrações dos compostos orgânicos nos diferentes extratos vegetais $\left(x ; \mathbf{m m o l ~ L}^{-1}\right)$

\begin{tabular}{cccc}
\hline $\mathbf{y}$ & Composto orgânico & Equação de regressão & $\mathbf{R}^{2}$ \\
\hline $\mathrm{pH} \mathrm{em} \mathrm{CaCl} 2$ & $\mathrm{AO}$ & $\hat{\mathrm{y}}=0,028 \mathrm{x}+4,796$ & $0,91^{* *}$ \\
& $\mathrm{LOH}$ & $\hat{\mathrm{y}}=0,413 \mathrm{x}+4,659$ & $0,85^{* *}$ \\
& $\mathrm{AOT}$ & $\hat{\mathrm{y}}=0,028 \mathrm{x}+4,796$ & $0,91^{* *}$ \\
$\mathrm{Al} \mathrm{trocável}$ & $\mathrm{AO}$ & $\hat{\mathrm{y}}=-0,019 \mathrm{x}+0,779$ & $0,94^{* *}$ \\
$\left(\mathrm{cmol}_{\mathrm{c}} \mathrm{dm}^{-3}\right)$ & $\mathrm{LOH}$ & $\hat{\mathrm{y}}=-0,292 \mathrm{x}-0,893$ & $0,98^{* *}$ \\
& $\mathrm{AOT}$ & $\hat{\mathrm{y}}=-0,028 \mathrm{x}+0,889$ & $0,96^{* *}$ \\
$\mathrm{H}+\mathrm{Al}$ & $\mathrm{AO}$ & $\hat{\mathrm{y}}=-0,087 \mathrm{x}+6,729$ & $0,78^{*}$ \\
$\left(\mathrm{cmol} \mathrm{dm}{ }^{-3}\right)$ & $\mathrm{LOH}$ & $\hat{\mathrm{y}}=-1,382 \mathrm{x}+7,309$ & $0,87^{* *}$ \\
$\mathrm{~A} \%$ & $\mathrm{AOT}$ & $\hat{\mathrm{y}}=-0,134 \mathrm{x}+7,304$ & $0,88^{* *}$ \\
& $\mathrm{AO}$ & $\hat{\mathrm{y}}=0,777 \mathrm{x}+33,688$ & $0,94^{* *}$ \\
& $\mathrm{LOH}$ & $\hat{\mathrm{y}}=12,043 \mathrm{x}+29,011$ & $0,98^{* *}$ \\
\hline
\end{tabular}

*: $p<0,05 \mathrm{e}^{* *}: \mathrm{p}<0,01$, por análise de regressão. 
pelos compostos orgânicos hidrossolúveis nos materiais vegetais e que os métodos utilizados foram efetivos na estimativa desses compostos orgânicos. As reações de complexação de ácidos orgânicos de baixo peso molecular que modificam o comportamento dos cátions polivalentes e amenizam a acidez no solo já foram descritas anteriormente (Hue et al., 1986; Noble et al., 1995; Miyazawa et al., 2002). A técnica potenciométrica com eletrodo seletivo de íons $\mathrm{Cu}^{2+}$ seria a mais indicada para estimar os compostos orgânicos hidrossolúveis em estudos referentes ao efeito desses compostos na neutralização do $\mathrm{Al}^{3+}$ e mobilização de $\mathrm{Ca}$ e $\mathrm{Mg}$ no solo, uma vez que os ácidos orgânicos estimados por essa técnica seriam os mais relevantes nas alterações químicas citadas, pela maior constante de estabilidade dos complexos organometálicos formados.

\section{CONCLUSÕES}

1. A aplicação de calcário e de extratos de resíduos vegetais promoveu a amenização do $\mathrm{Al}$ trocável, da acidez do solo e a mobilidade dos cátions básicos polivalentes até às subcamadas da coluna de solo.

2. Os resíduos de nabo forrageiro e de aveia foram mais eficientes na neutralização da acidez e mobilização de cátions polivalentes do que os resíduos de trigo, milho e soja.

3. Os compostos orgânicos dos extratos vegetais apresentaram correlação significativa com as alterações de $\mathrm{pH}, \mathrm{Al}$ trocável, $\mathrm{H}+\mathrm{Al}$ e a saturação por bases nas colunas de solo.

\section{LITERATURA CITADA}

AMARAL, A.S.; ANGHINONI, I. \& DESCHAMPS, F.C. Resíduos de plantas de cobertura e mobilidade dos produtos da dissolução do calcário aplicado na superfície do solo. R. Bras. Ci. Solo, 28:15-123, 2004.

CAIRES, E.F.; BLUM, J. \& BARTH, G. Alterações químicas do solo e resposta da soja ao calcário e gesso aplicados na implantação do sistema plantio direto. R. Bras. Ci. Solo, 23:275-286, 2003.

CASSIOLATO, M.E.; MEDA, A.R.; PAVAN, M.A.; MIYAZAWA, M. \& OLIVEIRA, J.C. Evaluation of oat extracts on the efficiency of lime in soil. Braz. Arch. Biol. Technol., 43:533$536,2000$.

DEVÊVRE, O.; GARNAYE, J. \& BOTTON, B. Release of complexing organic acids by rhizosphere fungi as a factor in Norway spruce yellowing in acidic soils. Mycol. Res., 100:1367-1374, 1996.
FRANCHINI, J.C.; GONZALEZ-VILA, F.J.; CABRERA, F.; MIYAZAWA, M. \& PAVAN, M. A. rapid transformations of plant water-soluble organic compounds in relation to cation mobilization in an acid Oxisol. Plant Soil, 231:55-63, 2001.

GONZALEZ-ERICO, E.; KAMPRATH, E.J.; NADERMAN, G.C. \& SOARES, W.V. Effect of depth of lime incorporation on the growth of corn on an Oxisol of Central Brazil. Soil Sci. Soc. Am. J., 43:1155-1158, 1979.

HUE, N.V.; CRADDOCK, G.R. \& ADAMS, F. Effect of organics acids on aluminum toxicity in subsoils. Soil Sci. Soc. Am. J., 50: 28-34, 1986.

JONES, D.L. Organic acids in the rhizosfere: A critical review. Plant Soil, 205:25-44, 1998.

LIU, J. \& HUE; N.V. Ameliorating subsoil acidity by surface application of calcium fulvates derived from common organic materials. Biol. Fert. Soils, 21:264-270, 1996.

MEDA, A.R.; CASSIOLATO, M.E.; PAVAN, M.A. \& MIYAZAWA, M. Alleviating soil acidity through plant organic compounds. Braz. Arch. Biol. Technol., 44:185-189, 2001.

MIYAZAWA, M.; PAVAN, M.A. \& FRANCHINI, J.C. Evaluation of plants residues on the mobility of surface applied lime. Braz. Arch. Biol. Technol., 45:251-256, 2002.

MOREIRA, S.G.; KIEHL, J.C.; PROCHNOW, L.I. \& PAULETTI, V. Calagem em sistema de semeadura direta e efeitos sobre a acidez do solo, disponibilidade de nutrientes e produtividade de milho e soja. R. Bras. Ci. Solo, 25:71-81, 2001.

NOBLE, A.D.; RANDALL, P.J. \& JAMES, T.R. Evalution of two coal-derived organic products in ameliorating surface and subsurface soil acidity. Eur. J. Soil Sci., 46:65-75, 1995.

OLIVEIRA, E. \& PAVAN, M.A. The control of soil acidity in no-till system for soybean production. Soil Till. Res., 38:47-57, 1996

PAVAN, M.A.; BLOCH, M.F.; ZEMPULSKI, H.D.; MIYAZAWA, M. \& ZOCOLER, D.C. Manual de análise química do solo e controle de qualidade. Londrina, IAPAR, 1992. 40p. (Circular, 76)

PAVAN, M.A. \& MIYAZAWA, M. Mobilização do calcário no solo através de resíduos da aveia. In: REUNIÃO DA COMISSÃO BRASILEIRA DE PESQUISA DA AVEIA, 18., Londrina, 1998. Anais. Londrina, IAPAR, 1998. p.72-79.

PAVAN, M.A. Movimentação do calcário no solo através de técnicas de manejo da cobertura vegetal em pomares de macieira. R. Bras. Frutic.,16:351-361, 1994.

YOUNG, S.D.; BACHE, B.W.; WELCH, D. \& ANDERSON, H.A. Analysis of the potentiometric titration of natural and synthetic poly-carboxylates. Soil Sci., 32:579-592, 1981.

ZIGLIO, C.M.; MIYAZAWA, M. \& PAVAN, M.A. Formas orgânicas e inorgânicas de mobilização do cálcio no solo. Braz. Arch. Biol. Technol., 42:257-262, 1999. 
